\title{
Photodisintegration of Ultra-High-Energy Cosmic Rays revisited
}

\author{
E. Khan ${ }^{1}$, S. Goriely ${ }^{2}$, D. Allard ${ }^{1}$, E. Parizot ${ }^{1}$, T. Suomijärvi ${ }^{1}$ \\ A. J. Koning ${ }^{3}$, S. Hilaire ${ }^{4}$ and M. C. Duijvestijn ${ }^{3}$ \\ ${ }^{1}$ Institut de Physique Nucléaire, $I_{2} P_{3}$-CNRS/Université Paris-Sud, 91406 \\ Orsay, France \\ ${ }^{2}$ Institut d'Astronomie et d'Astrophysique, ULB - CP226, 1050 Brussels, Belgium \\ ${ }^{3}$ Nuclear Research and Consultancy Group, P.O. Box 25, NL-1755 ZG Petten, \\ The Netherlands \\ ${ }^{4}$ Département de Physique Théorique et Appliquée, Service de Physique Nucléaire, \\ B.P. 12 - F-91680 Bruyères-le-Châtel, France
}

\begin{abstract}
Recent microscopic and phenomenological calculations of giant dipole resonances for $\mathrm{A} \leq 56$ nuclei are presented. The derived photodisintegration cross sections are exhaustively compared to the photonuclear data available to date. An accurate description of the data is found. Our new calculations are also compared with the previous and widely-used estimates of Puget, Stecker and Bredekamp. The present calculations also include all the possible paths down the nuclear chart. The impact on the photodisintegration of ultra-high-energy cosmic rays (UHECR) is illustrated for a Fe source with typical energies of $10^{20-21} \mathrm{eV}$. At energies around $10^{20} \mathrm{eV}$, the new cross sections are found to modify the UHECR photodisintegration rates. At energies around $10^{21} \mathrm{eV}$, it is recommended to solve a full reaction network to estimate the photodisintegration rate of the UHECR.
\end{abstract}

Key words:

\section{Introduction}

Cosmic-rays have been observed up to energies of $\sim 310^{20} \mathrm{eV}[1,2]$, which raises a double problem. First, one has to find an accelerator capable of reaching such ultra high energies. Second, it has been known for almost four decades that the interaction of the cosmic microwave background radiation (CMB) with ultra-high-energy cosmic rays (UHECR) should lead to a sharp decrease in their flux above energies around $10^{2} 0 \mathrm{eV}[3,4]$. In the case of protons, which has given rise to the most detailed investigations, this cut-off is due to 
pion photoproduction from CMB photons with energies above the reaction threshold in the proton rest frame. It has been proposed, however, that heavier nuclei could contribute significantly to the high energy part of the spectrum, as originally investigated by Puget, Stecker and Bredekamp (PSB) $[5,6]$. The propagation of nuclei in the intergalactic medium is also influenced by interactions with the background radiation fields, through photodisintegration reactions.

In the nucleus rest frame, at typical UHECR energies of $10^{19}-10^{21} \mathrm{eV}$, the CMB photons are boosted to energies in the range between a few hundreds of keV to a few hundreds of $\mathrm{MeV}$. The interaction process between the UHECRs and the CMB is dominated by the giant dipole resonance (GDR) at photon energies below $30-50 \mathrm{MeV}$, and to a lesser extent by the quasideuteron emission for intermediate energies (between $50 \mathrm{MeV}$ and $150 \mathrm{MeV}$ ) and the pion photoproductions at energies above $150 \mathrm{MeV}[5,7]$. In the original PSB model, two major approximations are performed to estimate the intergalactic UHECR propagation. The first one concerns the total photoabsorption cross section which is parameterized as a simple Gaussian function [5,6], abruptly cut below the theoretical reaction threshold. The second one is based on the use of a reduced reaction network, involving only one nucleus for each value of A, to estimate the time evolution of the UHECR composition. More precisely, assuming that the $\beta$-decay of the unstable nuclei produced by photodisintegration is always faster than the corresponding photoemission rate, a unique nuclear path is followed from the initial ${ }^{56} \mathrm{Fe}$ to the final protons [5].

The aim of this work is to study the impact of these two approximations on the properties of the UHECR photodisintegration, and to provide the scientific community with new accurate determination of the photoreaction rates. Reaction model developments allow now for an accurate and reliable systematic estimate of the photodisintegration rate. More specifically, photoreactions have been extensively studied in the field of nucleosynthesis, where phenomenological parameterizations of the photoabsorption cross sections have been optimized during the last decades, and large-scale microscopic predictions have also emerged $[9,10]$. New compilations of experimental photoabsorption data also help in defining the degree of accuracy of the present reaction models to predict the corresponding cross sections. Important progress has also been performed in the same field of nucleosynthesis to solve large reaction networks exactly in order to follow the time evolution of the composition of the material in given astrophysical sites. Similar tools can therefore be used in the field of UHECR to test the PSB approximation of a reduced network.

Section 2 describes improved calculations of the photodisintegration cross sections, using several phenomenological and microscopic descriptions of the dominant E1-strength function. To test the accuracy of such models, they are systematically compared with the available photonuclear data for nuclei with $A<56$, and with the PSB Gaussian parameterization. Section 3 is devoted to the study of the impact of the newly-determined photodisintegration cross section on the propagation of a ${ }^{56} \mathrm{Fe}$ source. The approximation of the original PSB reduced network is also tested in Sect. 3 by comparing the UHECR photodisintegration obtained by solving the full reaction network exactly. 


\section{Calculation of the photodisintegration cross sections}

To describe the changes in abundance of the heavy nuclei as a result of the interaction of the UHECR with the CMB, a nuclear reaction network including all interactions of interest must be used. The chosen set of nuclear species are coupled by a system of differential equations corresponding to all the reactions affecting each nucleus, i.e. mainly photodisintegrations and $\beta$-decays. The rate of change of the number density $N_{Z, A}$ of nucleus $(Z, A)$ with charge number $Z$ and mass number $A$ can be written as

$$
\begin{aligned}
\frac{d N_{Z, A}}{d t} & =N_{Z+1, A} \lambda_{\beta}^{Z+1, A}+N_{Z-1, A} \lambda_{\beta}^{Z-1, A} \\
& +N_{Z, A+1} \lambda_{\gamma, n}^{Z, A+1}+N_{Z+1, A+1} \lambda_{\gamma, p}^{Z+1, A+1}+N_{Z+2, A+4} \lambda_{\gamma, \alpha}^{Z+2, A+4} \\
& +N_{Z, A+2} \lambda_{\gamma, 2 n}^{Z, A+2}+N_{Z+2, A+2} \lambda_{\gamma, 2 p}^{Z+2, A+2}+N_{Z+4, A+8} \lambda_{\gamma, 2 \alpha}^{Z+4, A+8} \\
& +N_{Z+1, A+2} \lambda_{\gamma, n p}^{Z+1, A+2}+N_{Z+2, A+5} \lambda_{\gamma, n \alpha}^{Z+2, A+5}+N_{Z+3, A+5} \lambda_{\gamma, p \alpha}^{Z+3, A+5} \\
& -N_{Z, A}\left[\lambda_{\beta}^{Z, A}+\sum_{x} \lambda_{\gamma, x}^{Z, A}\right]
\end{aligned}
$$

where $\lambda_{\beta}^{Z, A}$ is the $\beta$-decay rate of nucleus $(Z, A)$ and $\lambda_{\gamma, x}^{Z, A}$ its photoerosion rate followed by the emission of a single neutron $(x=n)$, proton $(x=p)$ or $\alpha$-particle $(x=\alpha)$ or the emission of multiple particles such as $2 n, 2 p, 2 \alpha, n p, \ldots$, including all open channels for a given photon energy distribution.

The CMB photon density $\mathrm{n}(\epsilon)$ depends only on the UHECR Lorentz factor $\gamma=E / M c^{2}$ (where $\mathrm{E}$ is the UHECR energy, and $\mathrm{M}$ its mass) [6]. The calculations of the CMB density as a function of the photon energy $\epsilon$ in the nucleus rest frame show that photon energies overlap with the nuclear GDR for $\gamma$ ranging from $5.10^{9}$ to $10^{12}$. In the nucleus rest frame, the photodisintegration rate $\lambda_{\gamma, x}$ can be estimated from the cross section $\sigma_{\gamma, x}(\epsilon)$ by

$$
\lambda_{\gamma, x}=\int n(\epsilon) \sigma_{\gamma, x}(\epsilon) c d \epsilon
$$

where $c$ is the light speed.

All nuclei lighter than the seed nuclei and located between the valley of stability and the proton drip line must be included in the network. Under the most natural astrophysical assumptions, UHECRs are accelerated out of the ambient gas, possibly enriched in $\mathrm{Fe}$ close to neutron stars or depleted in metals (i.e. nuclei heavier than $\mathrm{H}$ ) if significant photodisintegration occurs during the acceleration stage itself. Therefore, if nuclei are indeed present among the UHECRs, it is expected that they typically include the most abundant elements found in the interstellar medium, i.e. essentially lighter than Fe. The interaction of UHECRs with the CMB is thus expected to include all possible nuclei resulting from the photodisintegration of the heaviest species and therefore involve all stable and neutron-deficient unstable isotopes with $A \lesssim 56$. 
One of the most complete compilation of photonuclear data is provided by the 2000 IAEA atlas (2000) [11]. Nevertheless, as far as elements of interest in the propagation of UHECR are concerned, only a limited set of photonuclear cross sections are known, namely the total photoabsorption cross section as a function of energy for about 10 nuclei and the integrated total photoabsorption cross section for no more than 16 nuclei. All the remaining rates must therefore be estimated on the basis of theoretical reaction models.

\subsection{The E1-strength function}

The uncertainties involved in any cross section calculation are not so much related to the model of formation and de-excitation of the compound nucleus itself, than to the evaluation of the nuclear quantities necessary for the calculation of the transmission coefficients. The total photon transmission coefficient characterizing the probability to excite by photoabsorption a compound nucleus excited state is obviously one of the key ingredients for the evaluation of the photoreaction rates. In the specific astrophysical conditions considered here, i.e for UHECR energies of $10^{19-21} \mathrm{eV}$, this function is dominated by the $E 1$ transition which is classically estimated within the Lorentzian representation of the GDR. Experimental photoabsorption data confirm the simple semi-classical prediction of a Lorentzian shape at energies around the resonance energy $E_{G D R}$. One the most widely used form of the E1-strength function is described by the Brink-Axel Lorentzian model $[12,13]$

$$
T_{E 1}\left(\varepsilon_{\gamma}\right)=\frac{8}{3} \frac{N Z}{A} \frac{e^{2}}{\hbar c} \frac{1+\chi}{m c^{2}} \frac{\Gamma_{\mathrm{GDR}} \varepsilon_{\gamma}^{4}}{\left(\varepsilon_{\gamma}^{2}-E_{\mathrm{GDR}}^{2}\right)^{2}+\Gamma_{\mathrm{GDR}}^{2} \varepsilon_{\gamma}^{2}},
$$

where $E_{G D R}$ and $\Gamma_{G D R}$ are the energy and width of the GDR, $m$ is the nucleon mass and $\chi \simeq 0.2$ is an exchange-force contribution to the dipole sum rule.

The Lorentzian description is known to be less satisfactory at energies away from the GDR peak, and in particular fails to describe the low-energy experimental data, namely the radiation widths and gamma-ray spectra [14,16]. Various improvements have been brought to the Lorentzian form, mainly by including an energy-dependence of the GDR width capable of modifying the low-energy behavior of the E1-strength [8,14-16]. For this reason, the photon transmission coefficient is most frequently described in the framework of the phenomenological Kopecky-Uhl generalized Lorentzian model [16]. In this approximation, the GDR width of Eq. (3) is replaced by an energy-dependent width of the form $\Gamma\left(\varepsilon_{\gamma}\right)=$ $\Gamma_{G D R}\left[\varepsilon_{\gamma}^{2}+4 \pi T^{2}\right] / E_{G D R}^{2}$, where $T$ is the nuclear temperature and equals zero in the case of photoabsorption reactions. This model is the most widely used for practical applications, and more specifically when global predictions are requested for large sets of nuclei. It also requires the determination of the GDR peak energy and width to be predicted from some underlying model for each nucleus. For practical applications, these properties are either taken directly from experimental compilations (e.g [11,17]) whenever available, or obtained from a droplet-type model [18] or some experimental systematics [17].

The phenomenological Lorentzian approach suffers, however, from shortcomings of various 
sorts, and most particularly its lack of reliability when dealing with exotic nuclei or energies away from the GDR peak. For this reason, models of the microscopic type have been developed which are hoped to provide a reasonable reliability and predictive power for the E1-strength function. Attempts in this direction have been conducted within models like the thermodynamic pole approach [17], the theory of finite Fermi systems or the Quasiparticle Random Phase Approximation (QRPA) [19]. The spherical QRPA model making use of a realistic Skyrme interaction has even been used recently for the large-scale derivation of the E1-strength function. In such models, mean field calculations such as Hartree-Fock BCS (HFBCS)[20] and Hartree-Fock Bogoliubov (HFB) [21] are performed in order to describe the nucleus ground state. On top of these calculations, the QRPA is used to describe in a microscopic way the GDR. The linear response theory allows to predict the dipole strength of the excited nucleus. The only input of these mean-field models is the nucleon-nucleon interaction, including the Skyrme part, and the pairing component. Details can be found in [22].

Global HFBCS+QRPA and HFB+QRPA calculations for practical applications were developed in $[9,10]$ and shown to predict the location of the GDR in close agreement with experimental data and also to reproduce satisfactorily the average resonance capture data at low energies. These aforementioned QRPA calculations have been performed for all the $8 \leq Z \leq 110$ nuclei lying between the two drip lines. Note that the latest HFB+QRPA calculation is using the BSk7 Skyrme nucleon-nucleon interaction, initially derived to reproduce at best the measured masses [23].

\subsection{The photodisintegration cross sections}

The photoreaction cross sections are estimated with the code named Talys [24] which takes into account all types of direct, pre-equilibrium and compound mechanisms to estimate the total reaction probability as well as the competition between the various open channels. The photoreaction cross section is estimated at energies up to $50 \mathrm{MeV}$. The calculation includes single particle (nucleons and alpha) as well as multi-particle emissions. All the experimental information on nuclear masses, deformation and low-lying states spectra is considered, whenever available. If not, global nuclear level formulæ, and nucleon and alpha-particle optical model potentials are considered to estimate the particle transmission coefficients and the nuclear level. Details on the codes and the nuclear physics input (ground state properties, nuclear level densities, optical potential) can be found in the above mentioned references $[25,24]$. These various nuclear inputs are known to affect the photodisintegration rates mainly around the corresponding threshold energies, but have a relatively lower im-

pact than the $\gamma$-ray strength function. To estimate the accuracy of the different approaches available for the evaluation of an E1-strength function, the four models presented in the previous sub-section are considered, namely the Lorentzian [13], the generalized Lorentzian [16], the HFBCS+QRPA [9] and the HFB+QRPA [10]. In addition to the E1 component, the $E 2, M 1$ and $M 2$ contributions are also included in the calculation of the $\gamma$-ray strength as prescribed by [17], but are not varied due to their lower impact in comparison with the 
GDR. The quasideuteron process is also neglected in the present work due to the limited photon energy range [7].

\subsection{Comparison with the photonuclear data}

The photoreaction cross sections estimated as explained above are now compared with available experimental data [11] for nuclei with $\mathrm{A} \leq 56$. It should be recalled that, even for stable nuclei, the data on such nuclei are scarce: a cross section measurement, such as $(\gamma, 1 \mathrm{nx})$ or $(\gamma, \mathrm{abs})$, is available at various energies for less than half of the stable nuclei with $12 \leq \mathrm{A} \leq 56$. Total photoabsorption cross sections around the GDR peak energy are available for 10 nuclei, while the integrated total photoabsorption cross section is known for 16 nuclei [11]. The exhaustive comparison performed to compare the predictions with the data is illustrated below.

To test the model predictions, we first consider the $(\gamma, 1 \mathrm{nx})$ cross sections, i.e the photodisintegration leading to a single neutron emission, but possibly also to other extra ejectiles. This set is of particular importance for our present study since the one-nucleon emission is the dominant process for photon energies in the $0-50 \mathrm{MeV}$ range. The model predictions based on four different prescriptions for the E1-strength function is compared in Figs. 1 and 2 with experimental data for 8 nuclei, namely ${ }^{13} \mathrm{C},{ }^{23} \mathrm{Na},{ }^{30} \mathrm{Si},{ }^{32} \mathrm{~S},{ }^{35} \mathrm{Cl},{ }^{39} \mathrm{~K},{ }^{51} \mathrm{~V}$ and ${ }^{55} \mathrm{Mn}$. Globally, the calculations are found to describe the data accurately, from light to heavy nuclei, even though a few isotopes still suffer from imperfect description, such as ${ }^{13} \mathrm{C}$. In most cases, however, the behaviour of the cross-section is remarkably well described, being confined within the experimental error bars over the whole energy range. For astrophysical applications, this degree of accuracy is quite satisfactory, especially in view of the remaining uncertainties pertaining to the origin of UHECRs (distribution of sources, injection spectra, initial composition) and to their propagation in extragalactic space, depending on the essentially unknown strength and topology of the magnetic effects.

In some cases, the QRPA prediction is found to overpredict the data, especially at high energies. This is the case for the HFB+QRPA prediction of the ${ }^{32} \mathrm{~S}(\gamma, 1 \mathrm{nx})$ reaction, or for the HFBCS+QRPA results for ${ }^{30} \mathrm{Si},{ }^{51} \mathrm{~V}$ and ${ }^{55} \mathrm{Mn}$. On average, both phenomenological Lorentzian approaches reproduce better the experimental data. In the case of ${ }^{13} \mathrm{C}$ and

${ }^{23} \mathrm{Na}$, both the microscopic calculations and the Lorentzian-based predictions agree with each other. It should however be recalled here that the Lorentzian formulas make use of the experimental total photoabsorption peak cross section, peak energy and width for the cases presented here, while the QRPA models do not.

In order to illustrate the accuracy of multi-nucleon photoemission predictions, the ${ }^{51} \mathrm{~V}(\gamma, 2 \mathrm{nx})$ measured cross section is compared to the four calculations in Fig. 3. The agreement is good and all models predict similar cross sections, except the HFB+QRPA model which overestimates the cross section. The errors on such measurement are relatively large and prevent one from considering this data as a strong constraint on the models. 

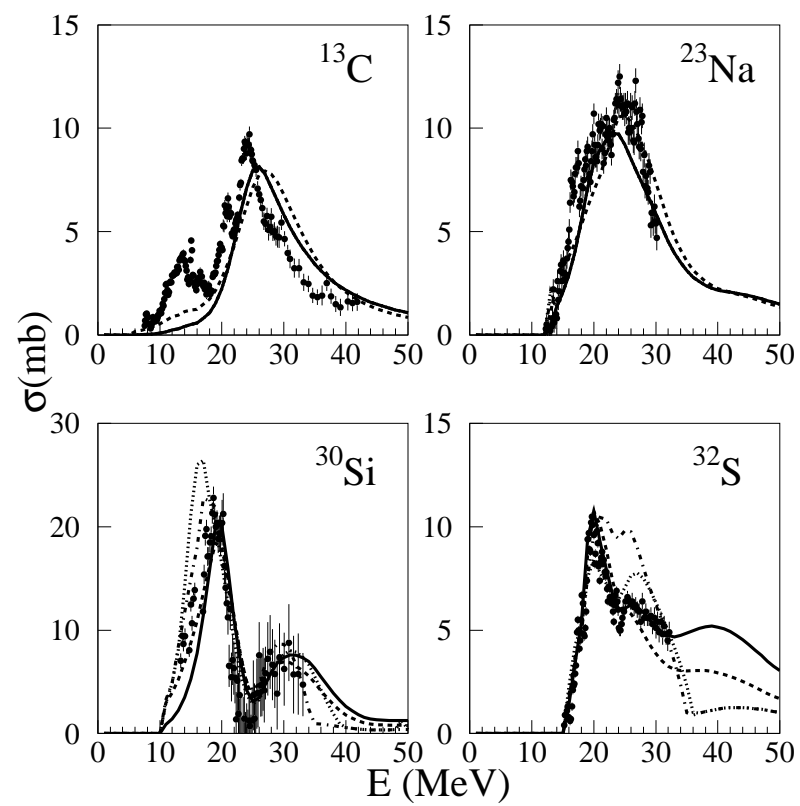

Fig. 1. Measured photoabsorption cross sections $(\gamma, 1 \mathrm{nx})$ state, compared to the predictions of the four models: Lorentzian (dashed line), generalized Lorentzian (solid line), microscopic HFBCS+QRPA (dotted line) and microscopic HFB+QRPA (dash-dot line)

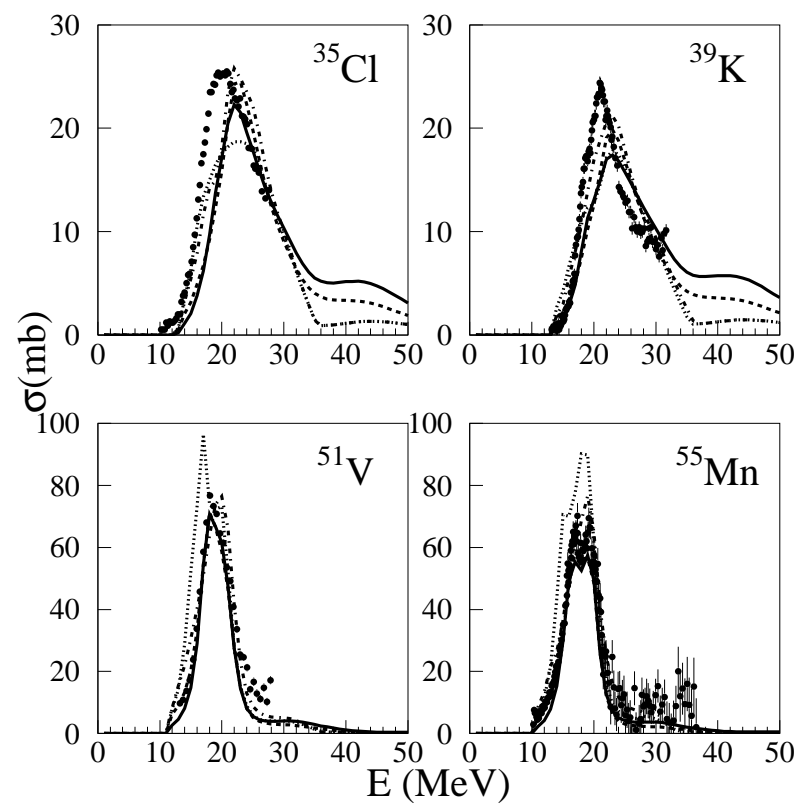

Fig. 2. Measured photoabsorption cross sections $(\gamma, 1 \mathrm{nx})$ state, compared to the predictions of the four models: Lorentzian (dashed line), generalized Lorentzian (solid line), microscopic HFBCS+QRPA (dotted line) and microscopic HFB+QRPA (dash-dot line) 


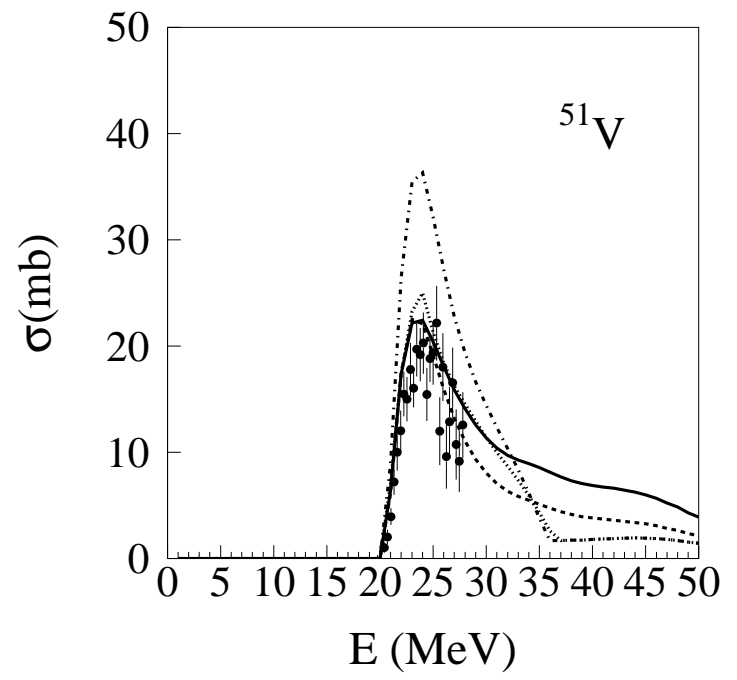

Fig. 3. Measured photoabsorption cross sections ${ }^{51} \mathrm{~V}(\gamma, 2 \mathrm{nx})$ compared to the predictions of the four models: Lorentzian (dashed line), generalized Lorentzian (solid line), microscopic HFBCS+QRPA (dotted line) and microscopic HFB+QRPA (dash-dot line)

The $(\gamma, \mathrm{p})$ cross sections provide complementary insight on the accuracy of the models. The integrated cross section is available for about 15 nuclei of interest [11]. The description of the data is found to be reasonable, and not to differ from one model to another significantly. An average agreement within a factor of 2 is obtained on the integrated cross section.

In view of the present comparison between the model predictions and the available experimental data, the generalized Lorentzian model is adopted for further calculations of the UHECR propagation. Since the nuclei of interest for the propagation of UHECR are located close to the valley of stability (see Sect. 3.2), experimental data provide a relatively accurate systematic determination of the GDR properties and no significant deviation from the phenomenological parameterization of the Lorentzian type is expected.

\subsection{Comparison with the PSB parameterization}

The generalized Lorentzian cross sections are now compared with the PSB Gaussian prescription. This comparison is made for total photoabsorption data. Although $(\gamma$,abs) cross sections are available for a limited number of nuclei (e.g. Ahrens et al. [26]), it allows a direct comparison of the $\gamma$-ray strength, regardless of the other nuclear inputs in the reaction calculation. The ${ }^{28} \mathrm{Si}$ and ${ }^{40} \mathrm{Ca}$ photoabsorption cross sections are compared in Fig. 4.

In their original work [5], PSB calculate the total photoabsorption cross section, relying on the total integrated cross section, available for about one third of the nuclei of interest 


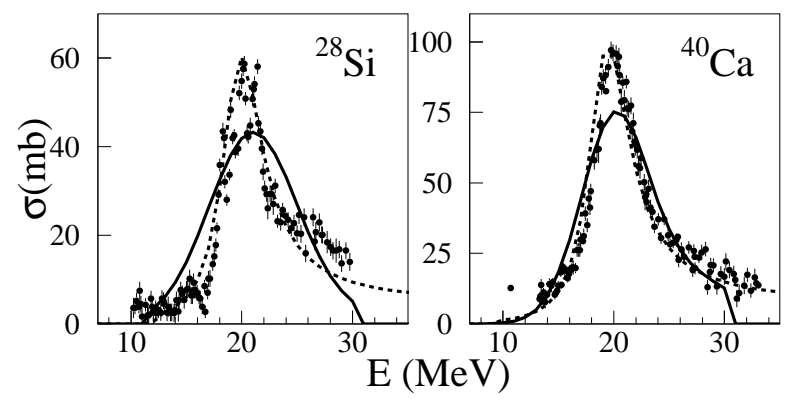

Fig. 4. Measured total photoabsorption cross sections, compared to the predictions of the PSB models (solid line) and the generalized Lorentzian (dashed line)

in the UHECR problem. This modelling of the GDR was performed up to an energy of $30 \mathrm{MeV}$. Though not strongly different from the Lorentzian calculations, it is clear that the PSB agreement with experimental data is not as good as the one obtained with the Lorentzian parameterization. This may be explained by two features of the PSB modelling. First the PSB parameterization assumes a Gaussian shape for the GDR, which can differ significantly from the experimentally-confirmed Lorentzian shape. Second, the width of the GDR is frequently overestimated by the PSB parameterization, compared to the data. An exhaustive comparison between the PSB and the Lorentzian parameterization for the $\sim 50$ nuclei used in the PSB model, shows also the general trend of a greater width (typically 2 $\mathrm{MeV}$ ) for the PSB parameterization. The large PSB width can lead to significant discrepancies, especially close to the one-neutron separation energy threshold located around 8 $\mathrm{MeV}$. The GDR contribution close to the neutron threshold plays an important role since it triggers the photodisintegration of UHECRs having just enough energy to be affected by the CMB photons while propagating throughout the universe. An accurate description of this threshold is thus necessary to derive the exact shape of the resulting turnover in the UHECR propagated spectrum.

The larger width also leads to a larger integrated value of the cross section between 0 and $30 \mathrm{MeV}$ than in the Lorentzian case. On the other hand the total integrated cross section between 0 and $50 \mathrm{MeV}$ is rather similar in both models since the Lorentzian prescription extends further up to $50 \mathrm{MeV}$ while the PSB Gaussian prescription is limited to energies below $30 \mathrm{MeV}$. Note that both models predict a similar location of the peak energy since they consider experimental information or experimentally-based systematics.

\section{Impact on the UHECR photodisintegration}

The intergalactic UHECR photodisintegration is calculated on the basis of the rates (Eq. 2) derived from the Talys cross sections described in Sect. 2. All stable and neutron-deficient unstable nuclei with $\mathrm{A} \leq 56$ are included in the reaction network (Eq. 1). The photodisintegration of $\mathrm{A} \leq 4$ nuclei are not considered. Only the interaction with the CMB is considered, 

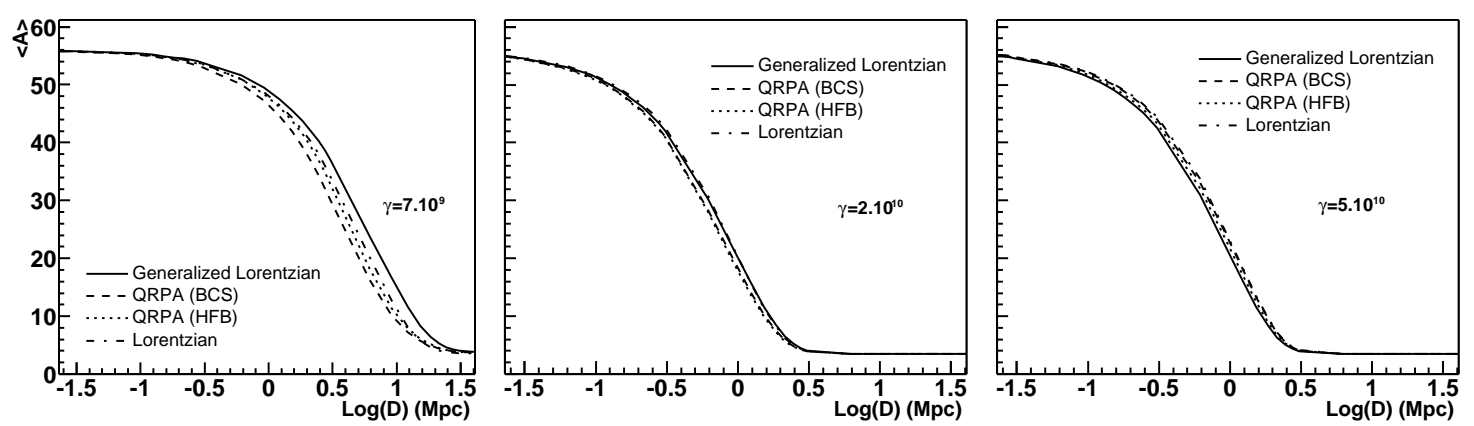

Fig. 5. Evolution of the average mass number $\langle A\rangle$ with respect to the distance of a ${ }^{56} \mathrm{Fe}$ source for three different Lorentz factors. Left: $\gamma=710^{9}\left(E=3.6 \times 10^{20} \mathrm{eV}\right)$; center: $\gamma=210^{10}$ $\left(E=10^{21} \mathrm{eV}\right)$; right: $\gamma=510^{10}\left(E=2.61 \times 10^{21} \mathrm{eV}\right)$.

the influence of the infrared background radiation being negligible in most cases of interest [6]. For illustrative purposes, we restrict ourselves to the case of a UHECR source made of ${ }^{56}$ Fe only. Other astrophysically relevant cases will be discussed elsewhere [27]. Note that a full propagation model for UHECRs (including pair production, statistical fluctuations, etc.) is not the purpose of this paper. Most recent calculations can be found, e.g., in [28,29].

\subsection{Impact of the photodisintegration cross sections}

Fig. 5 shows the evolution of the average mass number $\langle A\rangle$ as a function of the distance from the ${ }^{56} \mathrm{Fe}$ source, calculated with the four GDR prescriptions described in Sect. 2. For a given source distance, $\langle A\rangle$ is the average value of the calculated nuclei abundances:

$$
<A>=\frac{\sum_{i} N_{i} A_{i}}{\sum_{i} N_{i}}
$$

where $N_{i}$ is the number density given by Eq. (1). The full reaction network (Eq. 1) is solved at each time step, taking into account all the open photoemission channels, i.e $(\gamma, \mathrm{n}),(\gamma, \mathrm{p})$, $(\gamma, \alpha),(\gamma, 2 \mathrm{n}),(\gamma, 2 \mathrm{p}),(\gamma, 2 \alpha),(\gamma, \mathrm{np}),(\gamma, \mathrm{n} \alpha),(\gamma, \mathrm{p} \alpha)$. In other words, the abundance of each type of nucleus is derived by taking into account the contribution of all the production channels, from the source nucleus downwards the table of nuclides, with the appropriated weight derived according to the corresponding cross sections. The values obtained are thus equilibrium values, representing the composition which would result from the propagation of an infinite number of nuclei up to the time considered. For smaller UHECR samples, of course, Poissonian fluctuations of the numbers of each nuclear species are expected.

Three illustrative values of the Lorentz factors $\gamma$ are considered. The value $\gamma=710^{9}$ corresponds to an initial energy of the ${ }^{56} \mathrm{Fe}$ nucleus of $E=3.6 \times 10^{20} \mathrm{eV}$. In this regime, only the lowest energy part of the E1-strength overlaps with the photon density $\mathrm{n}(\epsilon)$. The photodisintegration rate is therefore sensitive to the position of the photoemission threshold located at the neutron separation energy $\left(S_{n} \sim 8 \mathrm{MeV}\right)$. The value $\gamma=210^{10}$ corresponds 
to a total energy of $E=10^{21} \mathrm{eV}$. The photon density is maximum around the neutron separation energy, and the photodisintegration rate is sensitive to the location of the GDR peak energy. Finally, the large value of $\gamma=510^{10}\left(E=2.61 \times 10^{21} \mathrm{eV}\right)$ corresponds to a regime mainly sensitive to the energy-integrated photodisintegration cross section.

Fig. 5 shows that iron nuclei with $\gamma=710^{9}$ propagate up to distances 10 times larger than in the higher energy cases, before being stripped from all their nucleons. This is due to the small overlap between the photon density and the photodisintegration cross section in the low- $\gamma$ case. In this regime, the distance of propagation is sensitive to the low-energy tail of the E1-strength function and the use of different prescriptions leads to significant differences in the propagation distance. In contrast, the high- $\gamma$ cases mainly depend on the GDR peak location or integrated photoabsorption, and for this reason the propagation distance is less sensitive to the photoreaction details. In addition, all calculations predict similar integrated photoabsorption cross sections since they all make use of the energyweighted sum rule to normalize the dipole strength [30]. The spread observed between the different curves in Fig. 5 reflects the impact of the remaining nuclear uncertainties on the UHECR propagation.

It should be noted that during propagation, the Lorentz factor of each fragment of the original nucleus remains essentially the same, since in a first approximation the total energy is evenly distributed among all nucleons. The whole propagation thus occurs at constant $\gamma$ and the interaction regime (at the threshold, around the peak or through the whole range of the cross section) remains the same along the fragmentation process. It should be reminded, however, that additional energy losses occur in real astrophysical situations, due to $\mathrm{e}^{+} \mathrm{e}^{-}$pair production as well as pion production. These important refinements are not included here because we focus on the specific influence of the nuclear cross sections, but they will be studied in detail in a forthcoming paper.

The UHECR mass distributions are displayed in Fig. 6 and show interesting features. The standard mass deviation is defined as :

$$
\sigma_{A}=\sqrt{\frac{\sum_{i} N_{i}\left(A_{i}-<A>\right)^{2}}{\sum_{i} N_{i}}}
$$

All the E1-strength parameterizations are seen to lead to similar predictions for the shape and magnitude of the standard deviation from the average mass number $\langle A\rangle$ as a function of the distance to the source. This overall mass distribution mainly depends on the relative competition between the various open photoemission channels, rather than on the absolute photoabsorption rate. This conclusion is valid irrespective of the energy of the source. At maximum spread, the average deviation from the mean value $\langle A\rangle$ reaches values as large as 7 mass units, which means that many different nuclei are found with comparable abundances as secondaries of the parent ${ }^{56} \mathrm{Fe}$ nucleus. In the specific case of $\gamma=710^{9}$, the mass distribution ranges from Ne to Fe isotopes at a distance $D=2.5 \mathrm{Mpc}$. 

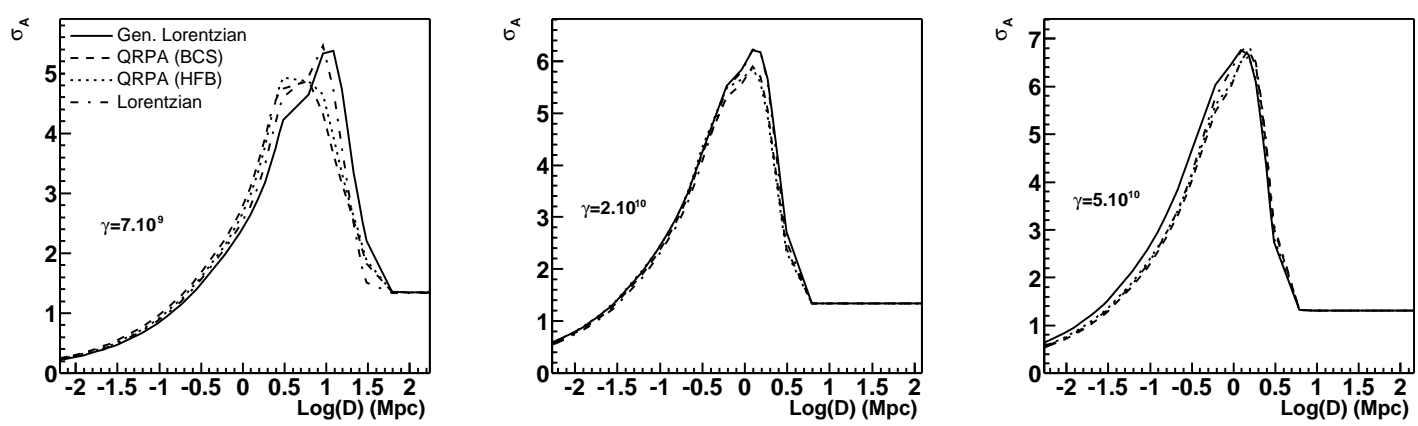

Fig. 6. Evolution of the standard deviation of the A distribution with respect to the distance of a ${ }^{56} \mathrm{Fe}$ source for three different Lorentz factors. Left: $\gamma=710^{9}\left(E=3.6 \times 10^{20} \mathrm{eV}\right)$; center: $\gamma=210^{10}\left(E=1.0 \times 10^{21} \mathrm{eV}\right)$; right: $\gamma=510^{10}\left(E=2.61 \times 10^{21} \mathrm{eV}\right)$.

\subsection{Photodisintegration path}

In Sect. 3.1, the UHECR propagation distance has been estimated making use of the full reaction network (Eq. 1). Previous calculations were based on the reduced PSB path illustrated in Fig. 7. In this approximation, only one stable isotope is considered per isobaric chain and the corresponding isobars (i.e. nuclei with the same A) are not affected by competitive channels. However, as shown in Fig. 7, about 85 nuclei are involved in the ${ }^{56} \mathrm{Fe}$ photodisintegration at $\gamma=2.10^{10}$ and numerous open channels including $\beta$-decay can compete (the Lorentz dilation of time allows $\beta$-unstable nuclei with half-lives of the order of the hour to survive over a Mpc scale, and thus have a chance to interact with a CMB photon). Most of the stable nuclei involved in the photodisintegration process have more neutrons than protons. Neutron emissions are therefore favoured and the corresponding unstable nuclei will $\beta^{+}$-decay towards the valley of stability. Note that we consider here that a given nucleus is involved in the reaction network if its calculated abundance amounts about $10 \%$ of the most produced one at any given time during the photodisintegration process.

Significant differences can therefore be expected between our new calculation (Fig. 5) and the original PSB results based on the reduced path and the Gaussian parameterization of GDR strengths. In particular, as seen in Fig. 7, for heavy nuclei $(A \geq 45)$, about $70 \%$ of the nuclei are shortcut by the simplified PSB path. For light nuclei $(\mathrm{A} \leq 45)$, less than $42 \%$ of the nuclei are bypassed.

Fig. 8 displays the average mass numbers $\langle A\rangle$ with respect to the source distance for the three $\gamma$ regimes. For each regime, three calculations are shown, namely (i) the full network calculation based on the generalized Lorentzian rates (solid line), (ii) the reduced PSB path calculation with the same generalized Lorentzian rates (dotted line), and (iii) the original PSB results based on the PSB path and Gaussian photodisintegration rates (dashed lines). The effect associated with the type of reaction network adopted is seen to be significant and to increase slightly with the UHECR energy. The simplified PSB path leads to a longer propagation distance with respect to the full network calculation, 


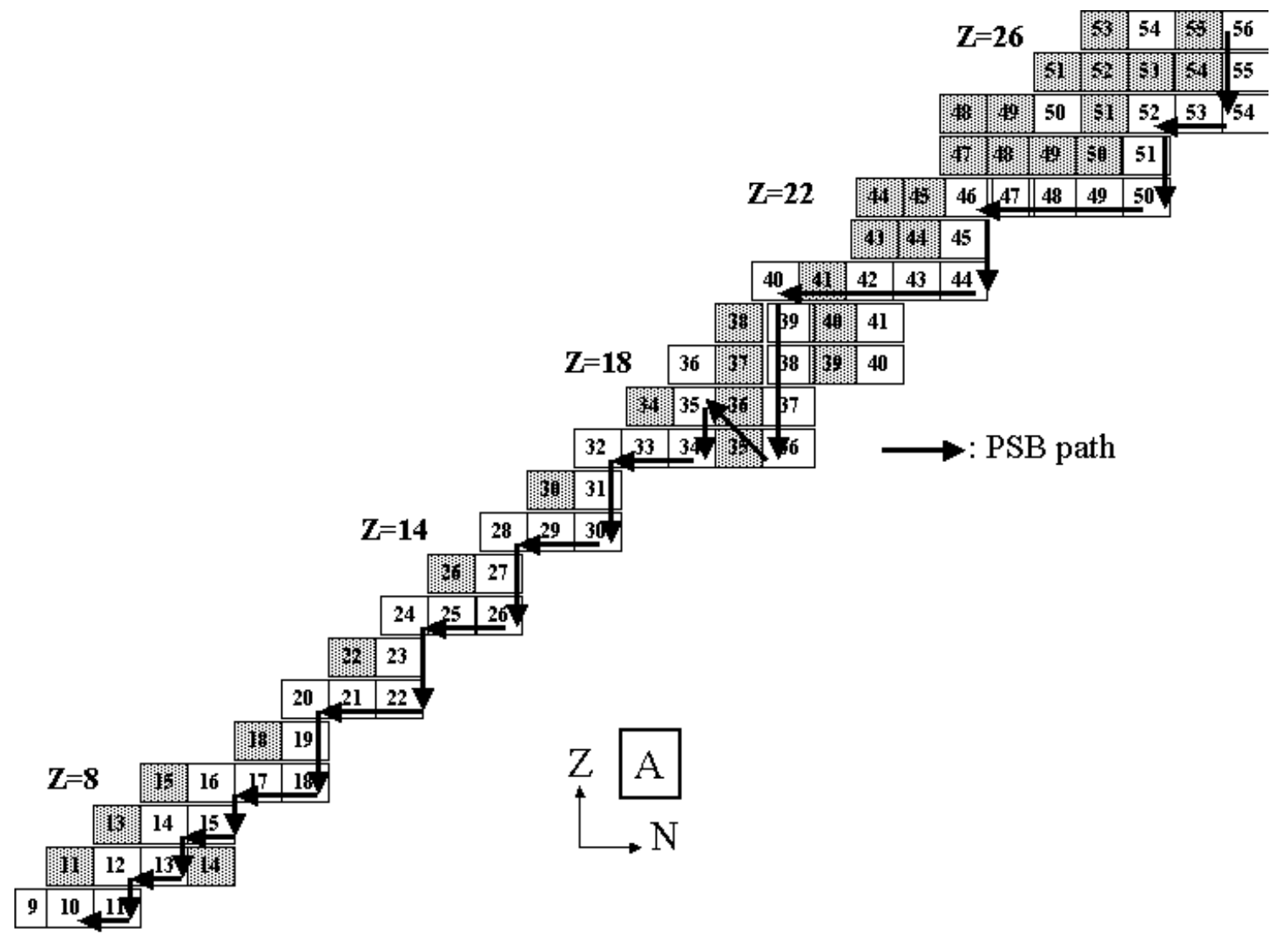

Fig. 7. Nuclei involved in the photodisintegration process of ${ }^{56} \mathrm{Fe}$ nuclei for $\gamma=2.10^{10}$. Unstable nuclei are in shaded squares, and the PSB path is indicated by the arrows. The mass number of each nucleus is written in the corresponding square.

independently of the nuclear input considered. This path effect is stronger for heavier than for lighter nuclei. For $\mathrm{A} \leq 45$, the curves show similar slopes. For the heavy species, the major differences stem from the large number of nuclei excluded from the PSB path, while the full reaction network calculations show that many isobars contribute to the nuclear flow. Within an isobaric chain, the photodisintegration cross section is usually larger for high Z-values, so that nuclei on the PSB path propagate up to large distances.

The differences between the dashed and dotted lines in Fig. 8 reflect the impact of the newly-determined photoreaction rates with respect to the widely used PSB rates. This comparison also confirms the previous conclusion that the cross section effect is attenuated at high energies due to similar integrated photoabsorption cross sections. However, the effect of the low-energy E1-strength around the threshold against particle emission remains significant, as seen in the low- $\gamma$ case. Both the path and the cross section have an impact on the propagation distance, whereas at high $\gamma$ values, the path effect is the only one to remain. 

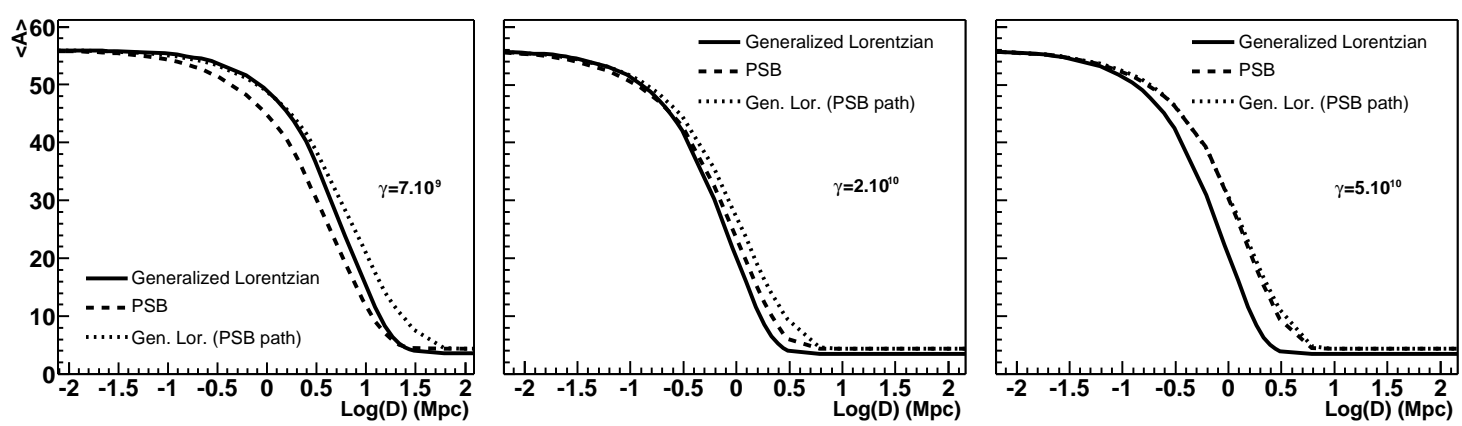

Fig. 8. Evolution of the average A with respect to the distance of a ${ }^{56} \mathrm{Fe}$ source for three different Lorentz factor. Left : $\gamma=710^{9}\left(E=3.6 \times 10^{20} \mathrm{eV}\right)$; center $: \gamma=210^{10}\left(E=1.0 \times 10^{21} \mathrm{eV}\right)$; right $: \gamma=510^{10}\left(E=2.61 \times 10^{21} \mathrm{eV}\right)$.

\section{Conclusions}

Four phenomenological and microscopic models have been used to predict the photodisintegration rates for nuclei with $\mathrm{A} \leq 56$ nuclei on the basis of the reaction code called Talys. The four models reproduce accurately the photonuclear data, namely the one-particle emission, two-particle emission, and total photoabsorption data. The original PSB description of the total photoabsorption cross section often overestimates the GDR width, implying a larger GDR contribution at low energies with respect to experimental data.

We studied in particular the case of a ${ }^{56} \mathrm{Fe}$ source and found that the new photoabsorption cross sections mainly modify the disintegration of UHECRs at relatively low Lorentz factors $\left(\sim 710^{9}\right)$, which is relevant to the shape of the cosmic-ray energy spectrum around the GZK suppression. The key ingredient proves to be a precise description of the GDR threshold. For higher Lorentz factors, details of the nuclear photoreaction rates do not impact much on the propagation distance. In contrast, it is found that solving a full reaction network can modify the results obtained with simpler nuclear paths, most particularly for the A $\geq 45$ nuclei. Large spreads in the mass distribution reaching \pm 7 mass units are obtained when use is made of the full reaction network.

The photodisintegration cross sections calculated with the generalized Lorentzian model are tabulated and made available to the scientific community via the nuclear astrophysics library at http://www-astro.ulb.ac.be. These include the $(\gamma, \mathrm{n}),(\gamma, \mathrm{p}),(\gamma, \alpha),(\gamma, 2 \mathrm{n}),(\gamma, 2 \mathrm{p})$, $(\gamma, 2 \alpha),(\gamma, \mathrm{np}),(\gamma, \mathrm{n} \alpha),(\gamma, \mathrm{p} \alpha) \ldots$ for all nuclei with $12 \leq A \leq 56$

The new approach to UHECR photodisintegration described in this paper will be applied to sensible astroparticle situations in forthcoming works, both using astrophysically motivated source compositions and taking into account the intergalactic magnetic fields.

Acknowledgments S.G. is FNRS Research Associate. This work has been performed within the scientific collaboration (Tournesol) between the Wallonie-Bruxelles Community and France. 


\section{References}

[1] H. Ayashida et al., Phys. ReV. Lett., 73 (1994) 3491

[2] D. J. Bird et al., Astrophys. J., 441 (1995) 144

[3] K. Greisen, Phys. Rev. Lett. 16 (1966) 748.

[4] G.T. Zatsepin and V.A. Kuzmin, Zh. Eksp. Teor. Fiz. Rev. 4 (1966) 114.

[5] J.L. Puget, F.W. Stecker and J.H. Bredekamp, Astr. Jour. 205 (1976) 638.

[6] F.W. Stecker and M.H. Salamon, Astr. Jour. 512 (1999) 521.

[7] J.P. Rachen, Ph.D. Thesis (1996), Friedrich-Whilhelms University (Bonn)

[8] S.Goriely, Phys. Lett. B436 (1998) 10.

[9] S.Goriely, E. Khan, Nucl. Phys. A706 (2002) 217.

[10] S.Goriely, E. Khan and M. Samyn, Nucl. Phys. A (2004), in Press.

[11] Photonuclear data for applications; cross sections and spectra, IAEA-Tecdoc-1178 (2000).

[12] D.M. Brink, Ph. D. Thesis, Oxford University (1955).

[13] P. Axel, Phys. Rev. 126 (1962) 671.

[14] C.M. McCullagh, M.L. Stelts, R.E. Chrien, Phys. Rev. C23 (1981) 1394.

[15] J. Kopecky, R. E. Chrien, Nucl. Phys. A468 (1987) 285.

[16] J. Kopecky, M. Uhl, Phys. Rev. C41 (1990) 1941.

[17] Reference Input Parameter Library, IAEA-Tecdoc (2003), in press

[18] W.D. Myers, W.D. Swiatecki, et al., Phys. Rev. C15 (1977) 2032.

[19] S.G.Kadmenskii, V.P. Markushev, V.I. Furman, Sov. J. Nucl. Phys. 37 (1983) 165.

[20] E. Khan and Nguyen Van Giai, Phys. Lett. B472 (2000) 253.

[21] M. Grasso, N. Sandulescu, Nguyen Van Giai, R. J. Liotta, Phys. Rev. C64 (2001) 064321.

[22] E. Khan, N. Sandulescu, Nguyen Van Giai and M. Grasso, Phys. Rev. C66 (2002) 024309.

[23] S. Goriely, M. Samyn, M. Bender and J.M. Pearson, Phys. Rev. C68 (2003) 054325.

[24] A.J. Koning, S. Hilaire and M.C. Duijvestijn, Proceeding of the International Conference on Nuclear Data for Science and Technology (Santa Fe, USA), 2004, in preparation

[25] A.J. Koning and J.P. Delaroche, Nucl. Phys. A713 (2003) 231. 
[26] J. Ahrens et al., Nucl. Phys. A251 (1975) 479.

[27] D. Allard et al., (2005) in preparation

[28] L. A. Anchordoqui, M. T. Dova, L. N. Epele, and J. D. Swain, Phys. Rev. D57 (1998) 7103

[29] L. N. Epele and E. Roulet, JHEP 10 (1998) 9

[30] P. Ring, P. Schuck, The nuclear many-body problem, Springer-Verlag (1980). 